\title{
$\mathrm{M}|\mathrm{R}| \mathrm{S}$ Internet Journal Nitride Semiconductor Research
}

\section{On the origin of the $2.8 \mathrm{eV}$ blue emission in p-type GaN:Mg : A time-resolved photoluminescence investigation}

\author{
F.Shahedipour ${ }^{1}$ and B.W.Wessels ${ }^{1}$ \\ ${ }^{1}$ Department of Materials Science and Engineering and Materials Research Center,
}

(Received Tuesday, June 19, 2001; accepted Friday, August 17, 2001)

The decay dynamics of the $2.8 \mathrm{eV}$ emission band in p-type $\mathrm{GaN}$ was investigated using timeresolved photoluminescence spectroscopy. The luminescence intensity decays non-exponentially. The decay dynamics were consistent with donor-acceptor pair recombination for a random distribution of pair distances. Calculations using the Thomas-Hopfield model indicated that recombination involves deep donors and shallow acceptors.

\section{Introduction}

In order to make GaN p-type, the material is doped with $\mathrm{Mg}$ to a concentration of greater than $1 \times 10^{19} \mathrm{~cm}^{-3}$. At this dopant concentration a strong blue luminescence ba nd peaked at $2.8 \mathrm{eV}$ is observed [1] [2] [3] [4]. Two models have emerged to describe the blue band luminescence, whose origin remains controversial. One model attributes the photoluminescence to donor-acceptor pair (DAP) recombination where the donor is s hallow and the acceptor is deep [4] [5] [6]. Alternatively, it has been proposed that DAP luminescence involves a deep donor and a shallow acceptor [1] [7]. Excitation intensity studies of the blue luminescence give strong support for the latter model [7]. The deep donor has been tentatively attributed to a nitrogen vacancy or nitrogen vacancy complex [8] [9]. Time dependent photoluminescence of this band has been initially studied. Nonexponential time decay has been observed that is attributed to a DAP transition [10] [11]. In the present study, transient photoluminescence spectroscopy was used to determine the nature of the defects responsible for the blue luminescence band in p-type GaN. From the time decay measurements and using the model of Thomas and Hopfield (T-H) for pair recombination, the acceptor involved is shown to be effective-mass-like, giving support to the deep donor-shallow acceptor model for this band.

\section{Experimental Results and Discussion}

For this study p-type GaN:Mg epitaxial layers were grown by atmospheric pressure metal organic chemical vapor deposition. Layers were $2 \mu \mathrm{m}$ thick with $20 \mathrm{~nm}$ thick GaN buffer layers. All the as-grown layers were semi-insulating. A post-deposition annealing in a nitrogen ambient at $700^{\circ} \mathrm{C}$ for 30 minutes was used to activate the $\mathrm{Mg}$ acceptors. Low temperature photoluminescence (PL) measurements (16 K) were used to characterize the defects in these materials. A $\mathrm{CW} \mathrm{He}-\mathrm{Cd}$ laser and a pulsed nitrogen laser (4 nsec pulse, $20 \mathrm{~Hz}$ repetition and a photon energy of $3.69 \mathrm{eV}$ ) were used for steady-state and time resolved PL measurements, respectively. A Spex grating monochromator with Hamamatsu photomultiplier tube was used, along with a series of neutral density filters to attenuate the excitation density. A closed cycle cryostat was employed for obtaining sample temperature in the range of $15 \mathrm{~K}-300 \mathrm{~K}$.

A typical low temperature steady-state PL spectrum of a p-type GaN:Mg sample is shown in Figure 1. Two main PL bands are observed, the dominant emission band centered at $2.8 \mathrm{eV}$ and a weaker ultraviolet (UV) band at $3.27 \mathrm{eV}$. The broad band centered at $2.8 \mathrm{eV}$ has been attributed to the contribution of DA pairs having different distances. The emission energy $E(r)$ is affected by the Coulomb interaction between acceptors and donors according to the following equation:

$$
E(r)=E_{g Q p}-\left(E_{A}+E_{D}\right)+\frac{e^{2}}{g r}
$$

where $\mathrm{E}_{\mathrm{gap}}$ is the band gap, $\mathrm{E}_{\mathrm{A}}$ and $\mathrm{E}_{\mathrm{D}}$ are the binding energy of acceptor and donor, $r$ is the separation between the donor and acceptor and $\varepsilon$ is the dielectric constant. Recombination involving close pairs contrib- 
utes to the high energy emission, whereas the more distant pairs are responsible for the low energy emission.

Time-resolved spectra of the $2.8 \mathrm{eV}$ blue emission was measured. Figure 2 shows the time-resolved spectra of this band for various time delays for excitation by the nitrogen laser. For DAP luminescence the recombination decay time of DAP luminescence will also depend on pair separation [12]. As is seen, the band shifts to lower energies as the time delay after the pulse excitation is increased. Pairs with a small separation will emit at high energy with short lifetime whereas the more distant pairs emit at lower energy with a longer decay time. The observed energy shift, as seen, is due to differences in the Coulomb interactions for close and distant pairs. The large red shift of approximately 200 $\mathrm{meV}$ of the band with time delay is an indication of its deep donor-acceptor transition nature [7].

Photoluminescence decay dynamics of the $2.8 \mathrm{eV}$ band have also been studied. Figure 3 shows time decay of this band. The decay was measured over three orders of magnitude from one hundred nanoseconds to 200 microseconds. A nominal decay time of one microsecond was observed that is consistent with the life time previously measured for the blue luminescence band [4] [10] [11]. Instead of a single decay time, the measured time decay is non-exponential. The observed decay dynamics can be explained in terms of the donor-acceptor pair recombination where the PL time decay depends on the detailed spatial distribution of donor and acceptor pairs.

The observed time dependence of intensity can be described by the DAP recombination model of Thomas and Hopfield (T-H) [12] [13] for a random distribution of pairs. According to this model, the time dependence of the emission intensity $\mathrm{I}(\mathrm{t})$ is given by:

$$
I(t)=\left\{4 \pi N \int_{0}^{\infty} W(r) \exp \left[-W(r) t r^{2} d r\right\} \times\left\{\exp \left[4 \pi N \int_{0}^{\infty}(\exp [-W(r) t]-1\} r^{2} d r\right]\right\}\right.
$$

where $N$ is the concentration of the majority constituent (either the donor or the acceptor), and $W(r)$ is the recombination rate for a donor-acceptor pair given by:

$$
W(r)=W_{\max } \exp \left(\frac{-2 r}{a_{0}}\right)
$$

where $W_{\max }$ is the transition probability in the limit of $r \rightarrow 0$ and $a_{0}$ is the Bohr radius of the weakly bound carrier. The theoretical model of the T-H model (Equation (2)) contains three independent parameters, namely, $W_{\text {max }}, N$ and the Bohr radius $\mathrm{a}_{0}$.
To fit the intensity data in Figure 3 using the T-H model, $W_{\max }$ and $N$ were used as fitting parameters. The Bohr radius was calculated using effective-mass approximation (EMA) since it has been shown that $\mathrm{Mg}$ behaves as a typical shallow acceptor [1] [7]:

$$
a_{A}=\frac{\hbar}{\sqrt{2 m_{h} E_{A}}}
$$

where $m_{h}$ is the effective mass of the hole in the valence band $\left(m_{h}=0.75 \mathrm{~m}_{\mathrm{e}}\right)$ and $E_{A}$ is the ionization energy. $E_{A}=0.2 \mathrm{eV}$ for an EMA acceptor in GaN [14]. The Bohr radius of the shallow $\mathrm{Mg}$ acceptor is calculated to be $5 \AA$ using the ionization energy of $0.2 \mathrm{eV}$. The best fit to the time decay data using Equation (2) and Equation (3) and the calculated value of the acceptor Bohr radius is shown in Figure 3 by the solid line. Excellent agreement between the calculated curve and the data is observed for times ranging from 100ns to $10 \mu \mathrm{s}$. The best fit was obtained for a majority constituent concentration $N$ of $(5 \pm 1) \times 10^{19} \mathrm{~cm}^{-3}$ and $W_{\text {max }}=(3$ $\pm 1) \times 10^{7} \mathrm{sec}^{-1}$. $W_{\max }$ is the capture rate for the DAP transition in the limit $r \rightarrow 0$. It should be noted that the measured value of $W_{\max }$ is of the same order of magnitude for DA recombination previously obtained for deep defects in $\mathrm{GaN}$ [13] [15]. The value of majority carrier obtained from the fit is consistent with the calculated acceptor concentration of $1 \times 10^{19} \mathrm{~cm}^{-3}$ obtained from Hall effect measurement of the hole concentration and assuming a compensation ratio of 0.1 .

\section{Conclusion}

Based on the time dependent decay measurements and analysis using the T-H model it is concluded that the blue luminescence results from DA recombination. The large red shift of the $2.8 \mathrm{eV}$ band with time delay is consistent with recombination involving a deep donor and a shallow effective mass acceptor. This is in agreement with the PL model proposed in previous studies for the blue luminescence band at $2.8 \mathrm{eV}$ [1] [7].

\section{ACKNOWLEDGMENTS}

The authors thank Dr. M. Reshchikov for his insightful discussions. This work was supported in part by NSF under Grant No. DMR-9705134 and NASA under Grant No.NAG5-6730.

\section{REFERENCES}

[1] U. Kaufmann, M. Kunzer, M. Maier, H. Obloh, A. Ramakrishnan, B. Santic, and P. Schlotter, Appl. Phys. Lett. 72, 1326 (1998). 
[2] M. Leroux, B. Beaumont, N. Grandjean, P. Lorenzini, S. Haffouz, P. Vennegues, J. Massies, P. Gibart, Mater. Sci. Eng. B 50, 97 (1997).

[3] B. Schineller, A. Guttzeit, P. H. Lim, M. Schwambera, K. Heime, O. Schon, M. Heuken, J. Cryst. Growth 195, 274 (1998).

[4] R. Seitz, C. Gaspar, T. Monterio, E. Pereira, M. Leroux, B. Beaumont, P. Gibart, Mater. Sci. Forum 258/263, 1155 (1997).

[5] A. K. Viswanath, E. Shin, J. I. Lee, S. Yu, D. Kim, B. Kim, Y. Choi, and C. -H. Hong, J. Appl. Phys. 83, 2272 (1998).

[6] E. Oh, H. Park, Y. Park, Appl. Phys. Lett. 72, 70 (1998).

[7] M. Reshchikov, C. G-. Yi, B. W. Wessels, Phys. Rev. B 59, 13176 (1999).

[8] K. J. Chang, S. -G. Lee, Mater. Sci. Forum 258/263, 1137 (1997).

[9] F. Shahedipour, B. W. Wessels, Appl. Phys. Lett. 76, 3011 (2000).

[10] Y. -H. Kwon, S. K. Shee, G. H. Gainer, G. H. Park, J. J. song, Appl. Phys. Lett. 76, 840 (2000).

[11] M. Godlewski, T. Suski, I. Grzegory, S. Porowski, J. P. Bergman, W. M. Chen, B. Monemar, Physica B 273-274, 39 (1999).

[12] D. G. Thomas, J. J. Hopfield, W. M. Augusuyniak, Phys. Rev. 140, A202 (1965).

[13] R. Y. Korotkov, M. A. Reshchikov, B. W. Wessels, Physica B 274, 80 (1999).

[14] M. Smith, G. D. Chen, J. Y. Lin, H. X. Jiang, A. Salvador, B. N. Sverdlov, A. Botchkarev, H. Morkoc, B. Goldenberg, Appl. Phys. Lett. 68, 1883-1885 (1996).

[15] D. M. Hoffmann, D. Kovalev, G. Steude, B. K. Mayer, A. Hoffmann, L. Eckey, R. Heitz, T. Detchprom, H. Amano, I. Akasaki, Phys. Rev. B 52, 16702 (1995).

\section{FIGURES}

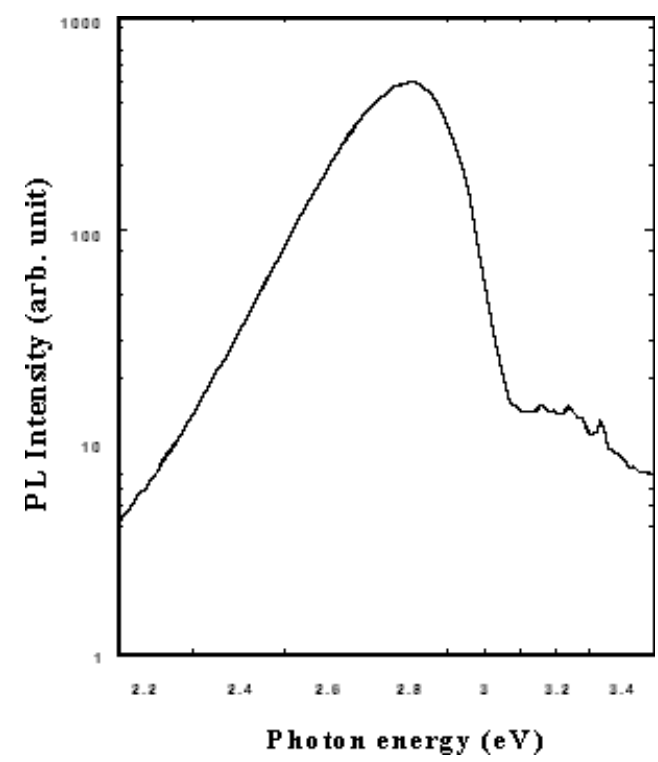

Figure 1. The steady-state PL spectrum of the $2.8 \mathrm{eV}$ band in $\mathrm{p}$ type $\mathrm{GaN}: \mathrm{Mg}$ measured at low temperature $(16 \mathrm{~K})$

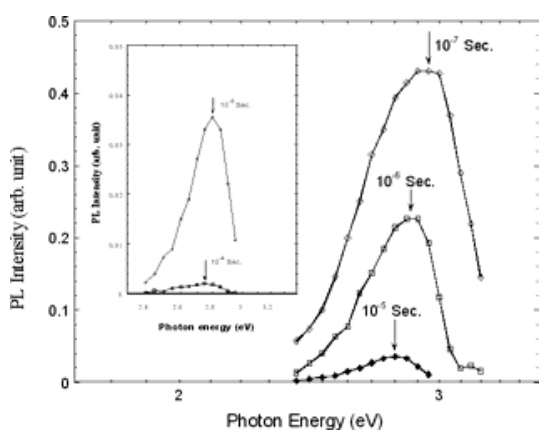

Figure 2. Time-resolved PL spectra of the $2.8 \mathrm{eV}$ emission band measured at low temperature for different time delays. The peak position at various delay times is indicated with an arrow. A shift to lower energies and narrowing of the band can be seen as the time delay is increased.

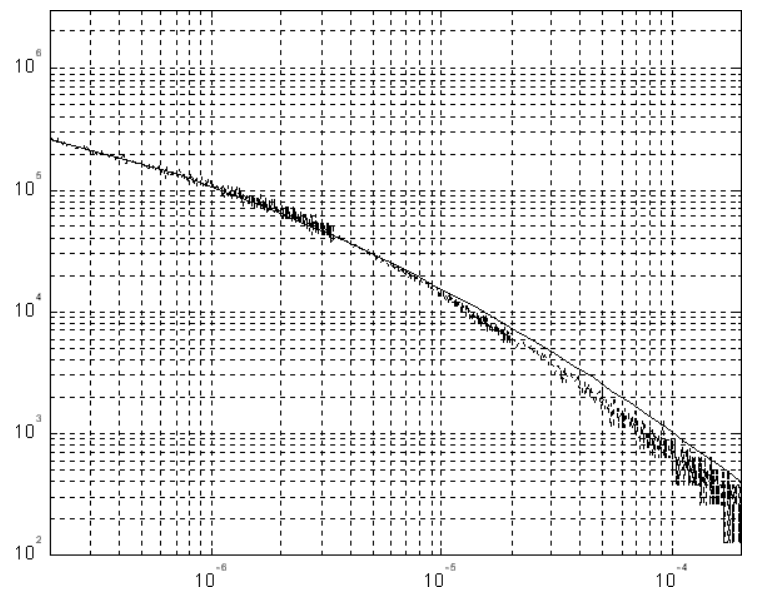

Figure 3. Photoluminescence intensity time decay for the 2.8 $\mathrm{eV}$ blue emission band plotted on a logarithmic scale. The calculated time decay from the T-H model for acceptor Bohr radius of $5 \AA$ is shown as indicated by the solid line. The fitting parameters are $\mathrm{N}=(5 \pm 1) \times 10^{19} \mathrm{~cm}^{-3}, \quad a_{A}=5 \AA$ and $W_{\max }=(3 \pm 1) \times 10^{7} \mathrm{sec}^{-1}$. 